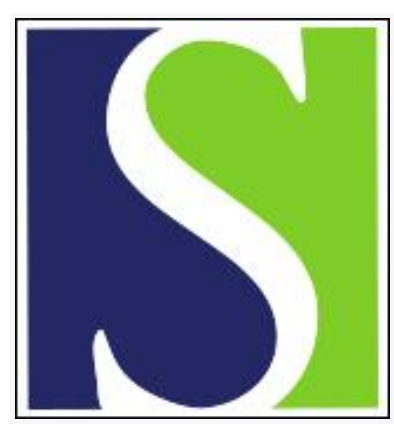

Scand J Work Environ Health 2002;28(6):367-369

https://doi.org/10.5271/sjweh.688

Issue date: Dec 2002

Improving exposure assessment requires measurements and modeling

by Schneider T

Affiliation: Scandinavian Journal of Work, Environment \& Health, Topeliuksenkatu 41 a A, Fl-00250 Helsinki, Finland.

Refers to the following texts of the Journal: 2002;28(6):371-385 1999;25(6):465-469 2001;27(5):354-357

Key terms: editorial; exposure assessment; modeling

This article in PubMed: www.ncbi.nlm.nih.gov/pubmed/12539796

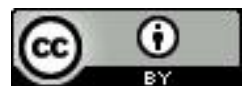




\section{Improving exposure assessment requires measurements and modeling}

In the proposal made by Vermeulen et al (1) for a systematic approach to dermal exposure assessment in occupational epidemiologic research in this issue of the Scandinavian Journal of Work Environment \& Health, the authors have transferred their insight gained from developing approaches to inhalation exposure assessment to the less developed field of dermal exposure assessment. The points made by the authors, when generalized, underline that the development of exposure assessment methodologies needs both measurement and modeling and that it is in the interplay between statistical and deterministic modeling, measurement, and other disciplines that progress can be made.

Exposure modeling at various levels of complexity are useful for (i) designing cost-effective strategies for measurements and the retrieval of historical data, (ii) analyzing, interpreting and reducing data, (iii) narrowing the exposure estimation variance obtained from measurements, (iv) drawing on information from other sources, (v) making retrospective exposure assessments, and (vi) translating data obtained in bench-scale tests to full-scale, to name a few. In the epidemiologic literature, the term "statistical model" is often used as opposed to "deterministic model". Deterministic models are generally built on equations based on mathematical principles, physics and chemistry, while statistical models are descriptive and are developed by fitting to observed data (2). In the statistical modeling of inhalation exposure, some general approaches have emerged, such as the use of mixed-effect models. The pool of results has had a major influence on our understanding of exposure variance components and their magnitude. Most mixedeffect and similar models have been fitted to the logs of measured concentrations. These models are constrained in the sense that they force all effects to act on exposure in a multiplicative way (upon backtransformation), and thus the results may not properly reflect the physics and chemistry governing exposure. Deterministic modeling is still at an exploratory stage. The lack of generally accepted schemes for decomposing exposure scenarios into exposure determinant classes hampers generalization, and thus it also hampers the general applicability of the results. The aim of deterministic modeling development should not be to develop the "grand unified deterministic exposure model" for chemical agents. If such a model could ever be developed, it would be intractable. Rather, a set of validated model-building blocks should be established that can be assembled into specific models.

Modeling is a way of thinking and reasoning about the exposure process, and models are used for the purpose of communicating (3). It is convenient to begin modeling by drafting a conceptual model and from there progress to a model for predicting exposure at a final level of complexity, which is determined by one's modeling skills and the type and amount of data available, or the amount that can be made available. There are many modeling approaches and many levels of abstraction. Single or multicompartment models are popular in the deterministic modeling of exposure to chemical agents. However, a wealth of other model types has been developed in engineering, social sciences, and like fields, and exposure assessors may have to look into such models to be able to deal with the new worklife scenario. This scenario requires rethinking of the entire exposure concept and approach to assessment (4). All types of exposure factors (physical, chemical, biological, psychosocial, and organizational) must be included for a complete characterization of exposure. Exposure is determined at three levels, all of which need to be investigated in the search for causes of effects on health and well-being and in the formation of recommendations for effective intervention. Societal systems and politics influence conditions on a national (macro) level, other factors affect circumstances on the company or department (meso) level, and, finally, some factors prevail at the individual (micro) level (5). The exposure determinants are nested 
within each other in several levels and may interact in a complex fashion. Recently investigators analyzing such scenarios have turned to qualitative methods in order to obtain insight into the situation as the workers see and experience them and define the underlying research questions to be investigated in targeted studies (5). Factors at the meso- and macrolevel that determine long-term average exposures, variance, and trends have received only little attention. An understanding of these factors would greatly add to our ability to document if, how much, and how fast workplace conditions improve.

Expert assessment of exposure has been, and still is, a common method in epidemiology. In earlier days, papers were published that did not specify the procedures used to reach the given exposure estimates, either because the procedures were "black boxes" (procedures existed in the minds of the assessors but were not visible to the observer) or there were no boxes at all. While it is now generally accepted that good expert assessment practice requires "white boxes", the very use of expert-based exposure assessment is under debate. True, many of the methods that served us well in the days of epidemiology, when risks were high, workplaces were homogeneous, and workforces were stable, should now be phased out. Should that include expert exposure assessment in general? No, at least not yet. In the area of dermal exposure, quantitative exposure data and standardized methods to quantify dermal exposures to many substances are lacking. Thus expert assessment will have a role to play in this area in the coming years. Vermeulen et al (1) urge that attempts be made to document and validate expert assessors' quantitative dermal exposure estimates. There should be no black boxes.

"Here are the historical documents-give me an exposure estimate" is just one way of (mis)using expert assessors. Their task can be structured by algorithms or by computer-aided coding, they can be asked to provide the prior probability distributions used in Bayesian estimation methods or input into deterministic models, and the like. In addition, statistical modeling contains elements of expert assessment in the formulation of models. These examples serve to show that expert assessors and modelers are companions. With a hint to the fact that many modelers have published deterministic models without proper validation, there are also "expert assessors" among the modelers.

There are many sources of bias in expert ratings, such as bias caused by the experts being "frame blind" (ie, they do not easily recognize their implicit assumptions and have difficulties in challenging their mental models), anchoring (ie, they hold to their original estimate and fail to adjust sufficiently as new facts are presented), being overconfident in their own estimates, and the like. Such sources of bias are well described in the literature, as are mathematical methods for scaling and averaging individual expert's ratings. However, much of this knowledge has not been transferred to the expert assessment of exposure to chemical agents. This situation could suggest that the full potential of expert exposure assessment has not yet been exploited.

More work is needed on how to combine the results of expert assessments with measured data mathematically or statistically and, more generally, on how to combine qualitative data and quantitative data. Wild et al's combining of measured exposures and expert-rated exposures in a statistical exposure model that used the expert ratings for constraining the estimates in the model (6) is an example of such possibilities.

While the distribution of dermal exposure over the body has been studied extensively, the evaluation of temporal and personal variability in dermal exposure measurements has been almost entirely absent. A recent compilation of dermal exposure measurements indicates that the total, within-worker, and between-workers geometric standard deviations are similar to those published for inhalation exposure (1). It is known from visualization studies that the individual execution of a given worktask can greatly affect inhalation exposure. Factors such as avoidance behavior and training come into play and raise the question of how well individual exposures can ever be modeled. In any case, there is a theoretical limit. Suppose that $N$ parameters are included in an exposure model and that the modeler is clever enough that the errors in model physics and chemistry decreases as $N$ increases. More and more of the 
variability can then be explained as $N$ increases. On the other hand, models need input, and, since all input data have some uncertainty, this error increases as $N$ increases. Thus the total model error does not decline to zero but, instead, has a minimum. In other words, there is an optimum degree of model complexity that minimizes the overall model error (7).

\section{References}

1. Vermeulen R, Stewart PA, Kromhout H. Dermal exposure assessment in occupational epidemiological research. Scand J Work Environ Health 2002;28:371-85.

2. Hornung RW, Greife AL, Stayner LT, Steenland NK, Herrick RF, Elliott LJ, et al. Statistical model for prediction of retrospective exposure to ethylene oxide in an occupational mortality study. Am J Ind Med 1994;25:825-36.

3. Fishwick PA. Simulation model design and execution: building digital worlds. Englewood Cliffs (NJ): Prentice Hall, 1995.

4. Hernberg S. Editorial. Scand J Work Environ Health 1999;25:465-9.

5. Hagberg M, Punnett L, Bergqvist U, Burdorf A, Härenstam A, Kristensen TS, et al. Broadening the view of exposure assessment. Scand J Work Environ Health 2001;27:354-7.

6. Wild P, Sauleau EA, Bourgkard E, Moulin J-J. Combining expert rating and exposure measurements: a random effect paradigm. Ann Occup Hyg 2002;46:479-87.

7. Hanna RS. Air quality model evaluation and uncertainty. J Air Poll Contr Assoc 1988;38:406-12. 


\section{Attention authors}

Beginning in 2003 the Journa1 will endorse the newest version of the "Uniform Requirements for Manuscripts Submitted to Biomedical Journals" (see: www.icmje.org).

Please note that, in their letters of submission, authors will now be required to add the following: (i) information on prior or duplicate publication or submission elsewhere of any part of the work, (ii) financial or other relationships that might lead to a conflict of interest, (iii) a statement that the manuscript has been read by all the authors, that the requirements for authorship have been met and that each author believes that the manuscript represents honest work, and (iv) any other information that may prove useful to the editor (eg, the type of article that the manuscript represents). Authorship credit should be based on (i) substantial contributions to the conception and design, or acquisition of data, or analysis and interpretation of data, (ii) drafting the article or revising it critically for important intellectual content, and (iii) final approval of the version to be published. Conditions i, ii, and iii must all be met. Acquisition of funding, the collection of data, or general supervision of the research group, by themselves does not justify authorship. To determine what comprises redundant or duplicate publication, see the "Requirements".

In addition, copies of the following statements will be required when necessary: (i) permission by all persons mentioned in the acknowledgments, (ii) permission to cite papers that have been accepted but not yet printed and also verification that they have been accepted for publication, (iii) permission to mention "unpublished observations" in the text, (iv) permission to use, and confirmation of the accuracy of, personal communications.

All hematologic and clinical chemistry measurements should be reported in the metric system in terms of the International System of Units.

Finally the Journal requests that authors send an IBM combatible disk with the manuscript in electronic form to help speed up the review process. The disk must be marked with the name of the author and the word-processing program used. A text file [rich text format (.rtf) or ASII (.txt) should also be included. Figures should be in tagged image format (.tif). 